\title{
Détection par GC-MS-MS dans les phanères de la prise illégale d'anabolisants stéroïdiens chez l'animal de production et chez les sportifs
}

\section{Gas chromatographic-tandem mass spectro- metric detection of anabolic steroids in hair for doping and meat quality controls}

Gilbert PEPIN $^{(1)}$, Yvan GAILLARD ${ }^{(1)}$, François VAYSSETTE ${ }^{(1)}$, Antoine BALLAND ${ }^{(2)}$, Françoise DOUCET ${ }^{(2)}$

(1) Laboratoire d'Expertise TOXLAB, 18, rue André del Sarte - 75018 PARIS Tél : 0142629886 - Fax : 0142573959

(2) Laboratoire ACE, 20, rue André del Sarte - 75018 PARIS

(Reçu le 8 décembre 1999 ; accepté le 12 janvier 2000)

\section{$R E ́ S U M E ́$}

Nous avons développé une méthode efficace, simple et sensible pour détecter la présence des stéroïdes anabolisants et de leurs esters dans les phanères. $100 \mathrm{mg}$ de poudre de phanères sont traités par du méthanol, par un bain à ultrasons pour l'extraction des esters, puis faire une lyse alcaline avec du NaOH $1 \mathrm{M}$ (de la soude) pour optimiser l'extraction des autres drogues. Les deux préparations liquides sont respectivement extraites à l'acétate d'éthyle, puis rassemblées. L'ensemble est purifié à l'aide d'une double extraction en phase solide sur cartouches NH2 et silice. Le résidu est dérivatisé au MSTFA. L'analyse est réalisée en chromatographie gazeuse couplée à un spectromètre de masse tandem (triple quadripôle). Généralement, l'ion parent choisi est l'ion moléculaire avec deux ions fils et ce pour chaque composé avec une énergie de collision se situant entre $-16 \mathrm{et}-21 \mathrm{eV}$. L'étalon interne est la nandrolone-d3 pour les dragues non estérifiées et le phényl propionate de testostérone pour les

\section{SUMMARY}

We have developed a powerful and simple sensitive method for testing hair for anabolic steroids and their esters. Hundred mg of powdered hair were treated with methanol in an ultrasonic bath for extraction of esters, then alkaline digested with I M NaOH for an optimum recovery of other drugs. The two liquid preparations were subsequently extracted with ethyl acetate, pooled, then finally highly purified using a twin solid phase extraction on amino and silica cartridges. Residue was derivatized with MSTFA prior to injection. Analysis was conducted by gas chromatography coupled to a triple quadrupole mass spectrometer. The generally chosen parent ion was the molecular ion while two daughter ions were selected for each compound with collision energies ranging from -16 to $-21 \mathrm{eV}$. Internal standards were nandrolone-d3 for non-esterified drugs and testosterone phenyl propionate for esters. The limits of detection calculated from an analysis of the blanks $(n=30)$ were 0.08 
esters. La limite de détection est calculée sur l'analyse des blancs $(n=30)$ soit $0,08 \mathrm{pg} / \mathrm{mg}$ pour la nandrolone; 6,20 pg/mg pour la boldénone; $0,07 \mathrm{pg} / \mathrm{mg}$ pour la méthyl testostérone ; $0,15 \mathrm{pg} / \mathrm{mg}$ pour l'éthinyl estradiol ; $2,10 \mathrm{pg} / \mathrm{mg}$ pour la méthandiénone ; $0,86 \mathrm{pg} / \mathrm{mg}$ pour le propionate de testostérone; $0,95 \mathrm{pg} / \mathrm{mg}$ pour le cypionate de testostérone; $1,90 \mathrm{pg} / \mathrm{mg}$ pour la nandrolone décanoate ; $3,10 \mathrm{pg} / \mathrm{mg}$ pour la testostérone décanoate et $4,80 \mathrm{pg} / \mathrm{mg}$ pour la testostérone undécanoate. Cette technique a été appliquée dans les contrôles des denrées animales et de dopage humain. Sur les 306 analyses réalisées sur des échantillons d'urines et de poils d'animaux provenant d'abattoirs, 20 ont été retrouvés positifs aux anabolisants dans les poils : 6 à la boldénone, 1 à la méthandiénone, 2 au propionate de testostérone et 11 à la méthyl testostérone. Dans le même temps, toutes les analyses effectuées sur les urines étaient négatives pour les anabolisants et leurs métabolites. Pour l'homme, à titre d'exemple, sur un échantillon de 18 sportifs, 2 ont été trouvés positifs aux anabolisants stéroüdiens dans les cheveux, alors que les résultats urinaires étaient négatifs pour les deux. Le premier cas est positif à la nandrolone et le second à l'undécanoate de testostérone.

\section{MOTS-CLÉS}

Phanère ; Dopage ; Anabolisants Stéroüdiens ; Esters de testostérone.

\section{Introduction}

\section{Utilisation illégale d'anabolisants stéroï- diens pour l'augmentation de produc- tion de viande de bétail}

L'utilisation d'anabolisants stérö̈diens et autres produits, type B-agonistes, permettant l'augmentation de la masse corporelle des animaux de rente a été interdite en Europe en 1989 pour faire face à l'abus de produits dont l'innocuité pour la santé humaine n'a jamais été prouvée. En effet, l'administration de ce type de substance permet un gain financier important pour les fraudeurs, augmentant le poids de l'animal (environ 10 à $25 \%$ ), améliorant le classement de la carcasse et diminuant les coûts de production. Les animaux sont ainsi plus beaux et moins chers à produire. L'association de différents produits est parfois très étonnante. On rapporte le cas en France d'une analyse au niveau du site d'injection révélant la présence simultanée de testostérone, testostérone cypionate, estradiol, estradiol benzoate, méthandriol, stanozolol et norethandrolone (1). Debruyckere et al. (2) rapporte trois cas suspects en Belgique en 1989 pour lesquels les métabolites de nandrolone ont été retrouvés chez des humains qui avaient consommé de la viande contaminée. Aux Etats-Unis, cependant, il n'a jamais été remis en question l'usage de cinq hormones : 3 physiologiques (testostérone, estradiol et progestérone) et 2 synthétiques (trenbolone et zéranol). Ces produits sont en vente libre sous forme $\mathrm{pg} / \mathrm{mg}$ for nandrolone, $6.20 \mathrm{pg} / \mathrm{mg}$ for boldenone, 0.07 $\mathrm{pg} / \mathrm{mg}$ for methyl testosterone, $0.15 \mathrm{pg} / \mathrm{mg}$ for ethinyl estradiol, $2.10 \mathrm{pg} / \mathrm{mg}$ for metandienone, $0.86 \mathrm{pg} / \mathrm{mg}$ for testosterone propionate, $0.95 \mathrm{pg} / \mathrm{mg}$ for testosterone cypionate, 1.90 $\mathrm{pg} / \mathrm{mg}$ for nandrolone decanoate, $3.10 \mathrm{pg} / \mathrm{mg}$ for testosterone decanoate and $4.80 \mathrm{pg} / \mathrm{mg}$ for testosterone undecanoate. Applications to meat quality control and doping control have been demonstrated. Of the 306 analyses realized based upon hair and urine sampling in slaughtering houses, 20 were positive for anabolic steroids in hair: 6 case for boldenone, 1 case for metandienone, 2 cases for testosterone propionate and 11 cases for methyl testosterone. In the meantime, urinalysis was always negative for these drugs or their metabolites. In a series of 18 sportsmen, 2 were tested positive for anabolic steroids in hair whereas urinalysis was negative for both of them. The first positive case was nandrolone and the second case concerned the identification of testosterone undecanoate.

\section{KEY-WORDS}

Hair ; Doping ; Anabolic steroids ; Testosterone esters.

d'implants qui sont placés sous la peau au niveau de l'oreille de l'animal. Le Codex alimentarius a fixé des limites pour les résidus des deux hormones de synthèse et aucune pour les 3 hormones naturelles. Malgré tout, des délais d'attente entre la dernière administration et l'abattage des animaux ont été fixés pour chacune des molécules.. Jusqu'à récemment, les méthodes de contrôles officielles étaient exclusivement basées sur la recherche urinaire de ces substances et/ou de leurs métabolites. En maîtrisant les données pharmacocinétiques (métabolisation et élimination) des ces molécules, ces contrôles s'avèrent simples à détourner par les fraudeurs. Considérant le potentiel des phanères sur la pérennité d'une molécule piégée dans la matrice kératinisée du poil, nous avons publié en juillet 1997 une première méthode (3) permettant la recherche des B-agonistes dans les poils. Cette publication a par la suite été confirmée par la publication d'une méthode officielle de référence LDH/LNR/98A en août 1998 [4]. En décembre 1998, une méthode officielle de référence $\mathrm{LDH} / \mathrm{LNR} / 98 \mathrm{~S}$ (5) a été publiée suivie de peu par notre publication (6) permettant la recherche des stéroïdes anabolisants dans le poil. Grâce à ces techniques, la combinaison des deux matrices (urine et poil) apparaît comme la seule procédure fiable pour lutter contre l'utilisation frauduleuse de ces substances interdites. En effet, l'urine apporte l'information d'une imprégnation récente ( 15 à 21 jours) alors que le poil permet quand à lui de détecter plus de six mois après l'administration de produits (3). 


\section{Utilisation illégale d'anabolisants stéroï- diens chez l'homme}

Le dopage consiste à faire usage d'une ou plusieurs substances ou procédés permettant une amélioration artificielle physique ou mentale de l'athlète $(7,8)$. L'usage des anabolisants stéroïdiens a été officiellement interdit au milieu des années 70 par les autorités sportives.

En 1974, le marché des esters de testostérone attire fortement 1'attention, car on sait de plus en plus que les esters sont indécelables dans les urines, leur métabolite étant la testostérone qui est physiologique. Les athlètes s'intéressent donc à l'utilisation des esters de testostérone comme moyen de dopage plusieurs semaines avant une compétition. La situation dans l'ex RDA exposée par Franke et Berendonk (9) et d'autres documents (1014), n'est pas une exception. Un médecin californien, Robert Kerr, a admis avoir prescrit des anabolisants stéroïdiens à plus de 20 médaillés olympiques au Jeux de Los Angeles en 1984 (15). Durant le Tour de France 1998, des éléments des enquêtes judiciaires relatées par la presse, puis des aveux publics de sportifs publiés dans deux livres $(16,17)$, ont montré pour ce sport que le dopage dans certaines équipes était plus ou moins généralisé et impliquait les médecins, les entraîneurs et les cyclistes eux-mêmes (18).

Il est bien connu qu'un temps de latence suffisant permet d'invalider les tests urinaires, ce qui explique le faible pourcentage de résultats positifs de la part des laboratoires accrédités par le Comité International Olympique (CIO). Des tests faits sur des cyclistes pris au hasard dans la région des Flandres en Belgique entre 1987 et 1994 ont aboutit à un taux de positivité de 7,8\% pour 4374 analyses (19). Par ordre décroissant de positivités aux tests urinaires, on trouve les amphétamines, l'éphédrine, la nandrolone et la testostérone. Lors des tests antidopages réalisés en 1988 par les 20 laboratoires accrédités par le CIO, sur 47.069 échantillons analysés, seulement 1153 cas positifs ont été trouvés (20). Le relatif petit nombre de cas positifs est directement lié à la matrice et aux techniques utilisées. Des technologies performantes telles que la spectrométrie de masse tandem (SM-SM) ou la spectrométrie de masse haute-résolution (SMHR) permettent de matérialiser une prise plus loin dans le passé. Cependant il n⿳y a pas eu d'augmentation du nombre de cas positifs, car les habitudes d'utilisation des agents dopants ont changé. Des cyclistes du Tour de France, par des témoignages spontanés et publiés, ont reconnu toujours prendre des anabolisants stéroïdiens, mais durant l'hiver, en arrêtant un ou deux mois avant les sélections et premières compétitions et poursuivant avec de l'hormone de croissance jusqu'aux épreuves (21-24). Parfois durant les compétitions, les précurseurs hormonaux de la testostérone sont consommés, notamment la déhydroépiandrostérone (DHEA), l'androstènedione et l'ansdrostènediol, ainsi que des hormones de croissance.

\section{Situation analytique actuelle}

\section{Analyses réalisées pour les bovins}

Gleixner et Meyer ont trouvé de l'estradiol et de la testostérone dans des poils de bovins par chromatographie liquide avec une détection immunoenzymatique (25). Les mêmes auteurs ont aussi trouvé de la méthyl testostérone et de l'éthynyl estradiol dans des poils d'animaux pour la production de viande par des tests immunoenzymatiques après une SPE et une purification par chromatographie liquide (26). Les veaux, au nombre de 8 , étaient nourris avec $3,5 \mu \mathrm{g}$ d'éthynyl estradiol et $35 \mu \mathrm{g}$ de méthyl testostérone par kilo de la masse du veau, deux fois par jour et pendant 10 jours. Les concentrations moyennes sont de $1,4 \mathrm{pg} / \mathrm{mg}$ pour l'éthynyl estradiol et de $2,7 \mathrm{pg} / \mathrm{mg}$ pour la méthyl testostérone.

En France la détection du clenbutérol, entre autres agonistes $\beta$-adrénergiques, et des anabolisants stéroïdiens dans les poils figurent désormais parmi les méthodes officielles du Ministère de l'Agriculture $(4,5)$.

\section{Analyses réalisées pour les humains}

La chromatographie en phase gazeuse couplée à la spectrométrie de masse (CPG-SM) représente la méthode de prédilection pour la recherche des anabolisants stéroïdiens dans les urines. L'utilisation d'une CPG-SM-SM à trappe d'ions a permis de détecter les traces de 23 stéroïdes (27). Cette technique très sensible permet de détecter et de confirmer des cas positifs qui n'auraient pu l'être par des méthodes de CPG-SM de routine. En diminuant la limite de détection, on augmente donc la période de détection. La détection et l'identification de la méthandiénone et du stanozolol par CPG-SMHR a permis à Schanzer et al. (28) de montrer que sur 116 cas positifs, 41 l'étaient en CPGSM et 75 en CPG-SMHR. La préparation des échantillons inclus une extraction sur phase solide (SPE) avec des cartouches de C18 suivie d'une hydrolyse enzymatique, puis d'une extraction liquide-liquide. La chromatographie liquide couplée à la spectrométrie de masse s'est révélée être un outil très efficace pour la détection de 17 esters de la testostêrone dans le plasma humain (29). Dans le cas d'un bodybuilder de 32 ans mort d'une crise cardiaque, il a été identifié dans ses cheveux de la méthandiénone, de l'épiméthandiénone, de la 6-ß-hydroxy-méthandiénone, de l'énantate de méthénolone, du décanoate de nandrolone, ainsi que différents esters de testostérone (propionate, isocaproate, phénylpropionate, énantate et décanoate) par CPGSMHR-SM (30). Kintz et al. rapportent la détection, par CPG-SM, de stanozolol, de nandrolone et de testostérone dans les cheveux de deux bodybuilders à des taux très élevés (31).

La loi n99-223 du 23 mars 1999 relative à la protection de la santé des sportifs et à la lutte contre le dopage (32) ne précise pas la nature des échantillons préle- 
vés lors des contrôles par les médecins agréés, mais précise qu'un décret en Conseil d'Etat déterminera les examens et les prélèvements autorisés. Nous espérons que les phanères seront retenus comme milieu utilisable en cas de contre-expertise.

Compte tenu du besoin et de l'intérêt des tests dans les phanères pour la recherche des anabolisants stérö̈diens chez l'animal et l'humain, nous avons développé en 1998, puis validé en 1999, une méthode puissante utilisant la CPG-SM-SM à triple quadripôle (6). L'autre intérêt majeur de l'analyse des phanères est la détection possible des esters de testostérone qui ne sont par ailleurs pas détectables dans les urines. Pour descendre à des niveaux de détections aussi bas que $1 \mathrm{pg} / \mathrm{mg}$, il est important d'obtenir des extraits aussi propres que possible en vue de diminuer le bruit de fond analytique. Bien qu'un peu complexe la méthode développée de préparation des échantillons permettant d'augmenter le rapport signal/bruit reste accessible à tous les laboratoires.

\section{Rappel sur l'incorporation des molé- cules dans les phanères et ses consé- quences sur les conditions d'extraction des anabolisants}

Les substances médicamenteuses, présentes dans la circulation générale suite à une consommation, sont incorporées dans les phanères par la voie endogène. Les molécules absorbées ou transférées par la transpiration le sont par la voie endogène-exogène. Et enfin, les molécules déposées par l'environnement extérieur (pollution, traitements cosmétiques) sont absorbées par la voie exogène.

Le mécanisme généralement proposé pour l'absorption des molécules dans les phanères par la voie endogène est une diffusion passive à partir du sang vers la base des follicules des poils et des cheveux. Les facteurs suivants favorisent alors l'accumulation des substances : le $\mathrm{pH}$ isoélectrique des cheveux étant proche de 6 matérialise le caractère acide de la matrice capillaire (33) et donc une bonne absorption des substances basiques ; un autre point important est l'interaction médicamenteuse avec la mélanine. Les mélanocytes sont des cellules hautement spécialisées, dont le $\mathrm{pH}$ intracellulaire a été estimé entre 3 et 5 (34). L'accumulation de molécules basiques y est donc favorisée.

Howells et al. (35) a étudié la fixation de plusieurs molécules (y compris le clenbutérol, la nandrolone, le diéthylstilbestrol et la trenbolone) à la mélanine sur des Sepia officinalis. Les molécules basiques et hydrophobes étaient les plus fortement liées. La désorption par l'éthanol était complète pour les molécules neutres mais partielle pour les molécules basiques. Ceci suggère que la fixation de ces dernières implique aussi un facteur ionique et qu'une sonication méthanolique sur un échan- tillon de poudre de cheveux pourrait être un prétraitement efficace pour extraire les anabolisants stéroïdiens.

\section{Matériel et méthodes Réactifs}

Le méthanol, le chloroforme, l'acétate d'éthyle, le dichlorométhane, le 2-mercaptoéthanol et le iodure d'ammonium sont fournis par Prolabo (Paris, France) ; la soude $\mathrm{NaOH} 1 \mathrm{M}$ vient de chez Carlo Erba ; le NMéthyl-(triméthylsilyl)-trifluoroacétamide (MSTFA) est fourni par Regis (Morton grove, IL, USA).

La boldénone, l'estradiol, l'éthynyl estradiol, la méthandiénone, la méthyl testostérone, la testostérone cypionate, la testostérone décanoate, la testostérone phényl propionate et la testostérone propionate nous ont été fournis par Sigma-Aldrich (Saint-Quentin Fallavier, France); la testostérone undécanoate vient de chez Organon (Bruxelles, Belgique) ; la nandrolone- $\mathrm{d}_{3}$ est un produit Cambridge Isotope Laboratories, CIL (Andover, MA, USA).

La solution d'étalon interne (E.I.) est un mélange à $1 \mu \mathrm{g} / \mathrm{ml}$ dans le méthanol de nandrolone- $d_{3}$ (E.I. $1_{1}$ pour les non-esters) et de testostérone phényl propionate (E.I.2 pour les esters).

L'agent de dérivatisation est un mélange de MSTFA-2mercaptoéthanol- $\mathrm{NH}_{4} \mathrm{I}(1000: 6: 2, \mathrm{v} / \mathrm{v} / \mathrm{m})$.

\section{Instrumentation}

Le chromatographe est le modèle 5890 séries II plus de chez Hewlett-Packard (Les Ulis, France) et il est équipé de l'injecteur automatique A200S de chez Finnigan Mat distribué par ThermoQuest (les Ulis, France). Le système de CPG est couplé avec un spectromètre de masse triple quadripôle TSQ 7000 de chez Finnigan Mat (Manchester, UK). La colonne capillaire de chromatographie est une CP SIL $8 \mathrm{CB}, 30 \mathrm{mx0}, 25 \mathrm{~mm}$ D.I., $0,25 \mu \mathrm{m}$ d'épaisseur de film de chez Chrompack (Les Ulis, France). L'hélium est le gaz vecteur utilisé. L'injecteur est à une température de $290^{\circ} \mathrm{C}$ et l'injection en mode splitless est pulsée par une montée de pression rapide maintenue pendant $0,75 \mathrm{~min}$. Le volume d'injection est de $2 \mu 1$. Les températures du SM sont de $300^{\circ} \mathrm{C}$ pour la ligne de transfert, $160^{\circ} \mathrm{C}$ pour la source et de $70^{\circ} \mathrm{C}$ pour les quadripôles. La température initiale du four est de $80^{\circ} \mathrm{C}$ pendant $2 \mathrm{~min}$. et augmente à raison de $15^{\circ} \mathrm{C} / \mathrm{min}$. jusqu'à $310^{\circ} \mathrm{C}$, température finale maintenue pendant $14 \mathrm{~min}$. Le SM est en mode d'ionisation par impact électronique. Le gaz de collision est l'argon à une pression de 1,7 mTorr. Les ions parents correspondent aux ions moléculaires des 14 composés (sauf pour la méthandiénone et l'éthynyl estradiol). Deux ions fils ont été choisis sur des critères de spécificité et d'abondance, et ils sont sélectionnés par le troisième quadripôle (Tableau). 
Pour les extractions sur phase solide, on utilise des cartouches de $\mathrm{NH}_{2}(500 \mathrm{mg} / 3 \mathrm{ml})$ et de Silice $(500 \mathrm{mg} /$ $3 \mathrm{ml}$ ) de chez IST distribué par Touzart et Matignon (Courtaboeuf, France), et une station d'extraction distribuée par Prolabo (Paris, France).

Le broyeur à boulet est le modèle EI 4000 de chez Kleco (Visalia, CA, USA).

\section{Échantillonnage}

Pour les bovins, les poils sont prélevés au moment de l'abattage avec $50 \mathrm{ml}$ d'urine. Les poils sont prélevés sur la tête de préférence. Aucune distinction n'est faite vis-à-vis de la couleur des poils pour les animaux bicolores.

Chez l'homme, la meilleure zone de prélèvement des cheveux se situe au niveau du vertex postérieur, car la variation de vitesse de pousse y est moindre. De plus, le cheveux s'altère moins avec l'âge et le sexe de la personne n'a pas d'influence. Les prélèvements doivent être au moins de 150-250 mg. S'il n'y pas de cheveux ou s'ils sont trop courts, les poils pubiens ou axillaires seront alors prélevés.

Le problème d'une contamination externe est très important surtout pour les drogues. L'approche est bien évidemment différente pour les anabolisants stéroïdiens qui se prennent par voie orale ou autre voie thérapeutique sous présentation pharmaceutique normale. Selon les recommandations de la Society of Hair Testing, notre procédure de lavage comprend un lavage de 2 min. au méthanol suivi de 2 lavages à l'eau chaude pendant 3 min. et enfin par 2 lavages de 3 min. au dichlorométhane $(36,37)$. Les échantillons sont ensuite finement coupés aux ciseaux et broyés.

\section{Prétraitement des échantillons (iden- tique qu'ils soient d'origine bovine ou humaine)}

Les anabolisants stéroïdiens non-esters sont stables dans des conditions alcalines fortes; une digestion par de la soude molaire semble être alors un prétraitement efficace de l'échantillon pour avoir le meilleur recouvrement des molécules recherchées à partir des poils. Malheureusement, les esters de testostérone et de nandrolone sont eux dans ces conditions irrémédiablement hydrolysés. L'extraction des stéroïdes à partir des phanères sera alors divisée en deux étapes.

Une masse $100 \mathrm{mg}$ de poudre de phanères est mise dans un tube vissé avec $10 \mu \mathrm{l}$ de solution d'étalons internes à $1 \mu \mathrm{g} / \mathrm{ml}$ et $1,5 \mathrm{ml}$ de méthanol. Le tube vissé est alors déposé dans un bain ultrasonique pendant 2 heures à $50^{\circ} \mathrm{C}$. Après une centrifugation, la phase méthanolique (avec les esters) est évaporée sous un courant d'air sec à $60^{\circ} \mathrm{C}$. La poudre restante est digérée par ajout de $2 \mathrm{ml}$ de $\mathrm{NaOH} 1 \mathrm{M}$ pendant $15 \mathrm{~min}$. à $80^{\circ} \mathrm{C}$; cette seconde étape permet d'extraire au mieux les anabolisants stêroïdiens non-esters.

\section{Préparation des échantillons}

\section{Extraction liquide-liquide}

Le résidu sec issu de la phase méthanolique est reconstitué avec $2 \mathrm{ml}$ d'eau distillée et de $100 \mu \mathrm{lde} \mathrm{NaOH} 0,1 \mathrm{M}$. L'addition de $2 \mathrm{ml}$ d'acétate d'éthyle permet une extraction liquide-liquide efficace des composés qui nous intéressent par une agitation douce du tube pendant 10 min. Après une centrifugation, la phase organique est déposée dans un autre tube propre. Cette extraction

Tableau I : Anabolisants stéroïdiens ey de leurs paramètres spectrométriques.

\begin{tabular}{|c|c|c|c|c|}
\hline Composé & Ion parent $(\mathbf{m} / \mathbf{z})$ & Energie de collision (eV) & $1^{\text {er }}$ ion fils $(\mathrm{m} / \mathrm{z})$ & $2 \mathrm{~d}$ ion fils $(\mathrm{m} / \mathrm{z})$ \\
\hline Nandrolone-d $\mathrm{d}_{3}\left(\right.$ E.I. $\left._{1}\right)$ & 421,2 & -21 & 133 & 287 \\
\hline Nandrolone & 419,2 & -21 & 182 & 194 \\
\hline Estradiol & 416,2 & -21 & 129 & 285 \\
\hline Testostérone & 432,2 & -21 & 209 & 301 \\
\hline Boldénone & 358,1 & -16 & 122 & 147 \\
\hline Méthyl testostérone & 446,2 & -18 & 301 & 356 \\
\hline Ethynyl estradiol & 425,2 & -18 & 193 & 231 \\
\hline Méthandiénone & 282,1 & -16 & 122 & 161 \\
\hline Testostérone propionate & 416,3 & -18 & 209 & 401 \\
\hline Testostérone cypionate & 484,3 & -21 & 209 & 469 \\
\hline Nandrolone décanoate & 500,3 & -21 & 182 & 194 \\
\hline Testostérone décanoate & 514,3 & -21 & 209 & 499 \\
\hline Testostérone phényl propionate (E.I.2) & 492,3 & -21 & 209 & 477 \\
\hline Testostérone undécanoate & 528,4 & -21 & 209 & 513 \\
\hline
\end{tabular}


liquide-liquide est effectuée en parallèle sur les phanères digérés par la soude. Les deux fractions d'acétate d'éthyle sont regroupées dans le même tube.

\section{Extraction liquide-solide sur des cartouches de $\mathrm{NH}_{2}$}

La purification des stéroïdes se fait par fixation des impuretés ionisées sur des colonnes amines (38). Les cartouches sont activées avec $3 \mathrm{ml}$ d'acétate d'éthyle. Les échantillons (les 2 fractions d'acétate d'éthyle réunies) sont alors déposés sur les cartouches, mais l'éluat contenant les stérö̈des est immédiatement récupéré, car ces composés ne sont pas retenus par la phase stationnaire. Une élution supplémentaire est réalisée avec $1 \mathrm{ml}$ d'acétate d'éthyle. L'évaporation est faite sous un courant d'air sec à chaud.

\section{Extraction liquide-solide sur des cartouches de silice.}

Le résidu est reconstitué par $1 \mathrm{ml}$ de chloroforme. Après l'activation des colonnes de silice par $3 \mathrm{ml}$ de chloroforme, les esters de testostérone et de nandrolone sont directement récoltés après le dépôt de l'échantillon sur la colonne. Une élution avec $1 \mathrm{ml}$ de chloroforme est effectuée. Une dernière élution par $2 \mathrm{ml}$ de chloroforme-acétate d'éthyle $(3: 1, \mathrm{v} / \mathrm{v})$ est réalisée afin de récupérer les stéroïdes non-esters. D'autres impuretés polaires sont ainsi retenues par la phase stationnaire.

\section{Dérivatisation}

Quelques anabolisants stéroïdiens n'ont pas un "bon" comportement chromatographique principalement dû à la présence de groupes hydroxyles et cétoniques dans leur structure [39]. Parmi les nombreux réactifs de dérivatisation des groupements hydroxyles, le MSTFA apparaît être celui de choix pour une triméthylsilylation (dérivé TMS). La présence d'un catalyseur est toutefois recommandée pour une totale dérivatisation : $\mathrm{NH}_{4} \mathrm{I}$ avec un agent réducteur (le 2-mercaptoéthanol). La colonne analytique doit être uniquement dédiée à l'analyse des dérivés TMS qui pourraient altérer le comportement des autres dérivés.

\section{Résultats}

\section{Application au contrôle de l'utilisation d'anabolisant chez l'animal de rente}

Dans le cadre de l'établissement d'un état des lieux de l'utilisation d'anabolisants dans le cheptel des bovins français, un comité interprofessionnel d'éleveurs a décidé de faire une série d'analyses, poils et urines, sur des animaux " douteux " présélectionnés par la conformation visuelle de leur carcasse qui laisse à penser que l'animal a reçu un traitement aux anabolisants. L'essai a été entrepris à partir de prélèvements effectués en abattoir dans toute la France sur 306 animaux. Des prélèvements de poils et d'urines ont été réalisés pour chaque animal. Les résultats positifs retrou vés dans le poil sont de 37, répartis de la façon suivante : les $ß$-agonistes avec le clenbutérol dans 17 cas, les hormones stérö̈diennes avec la méthyl testostérone dans 10 cas, la testostérone propionate dans 2 cas, la méthandiénone dans 1 cas et la boldénone dans 6 cas, et enfin le mélange ß-agonistehormone avec 1 cas de clenbutérol-méthyl testostérone.

Cela correspond à un pourcentage sur cette étude de $12,1 \%$ sur des bovins suspects présélectionnés. Les résultats positifs retrouvés dans les urines sont de 2 et uniquement sur les animaux positifs au clenbutérol. D'autres études (environ 200 cas) ont été réalisées dans le cadre d'affaires à caractères judiciaires où des résultats identiques en terme de recouvrement entre les urines et les phanères ont été mis en évidence. Les molécules stéroïdiennes les plus fréquemment retrouvées sont la méthyl testostérone (fig.1), le décanoate de testostérone et la nandrolone. La molécule $\beta$-agoniste la plus fréquemment retrouvée est le clenbutérol. L'ensemble de ces résultats matérialise d'une manière évidente, l'intérêt de l'association poil et urine dans la recherche d'une utilisation frauduleuse de substance anabolisante interdite d'utilisation en Europe.

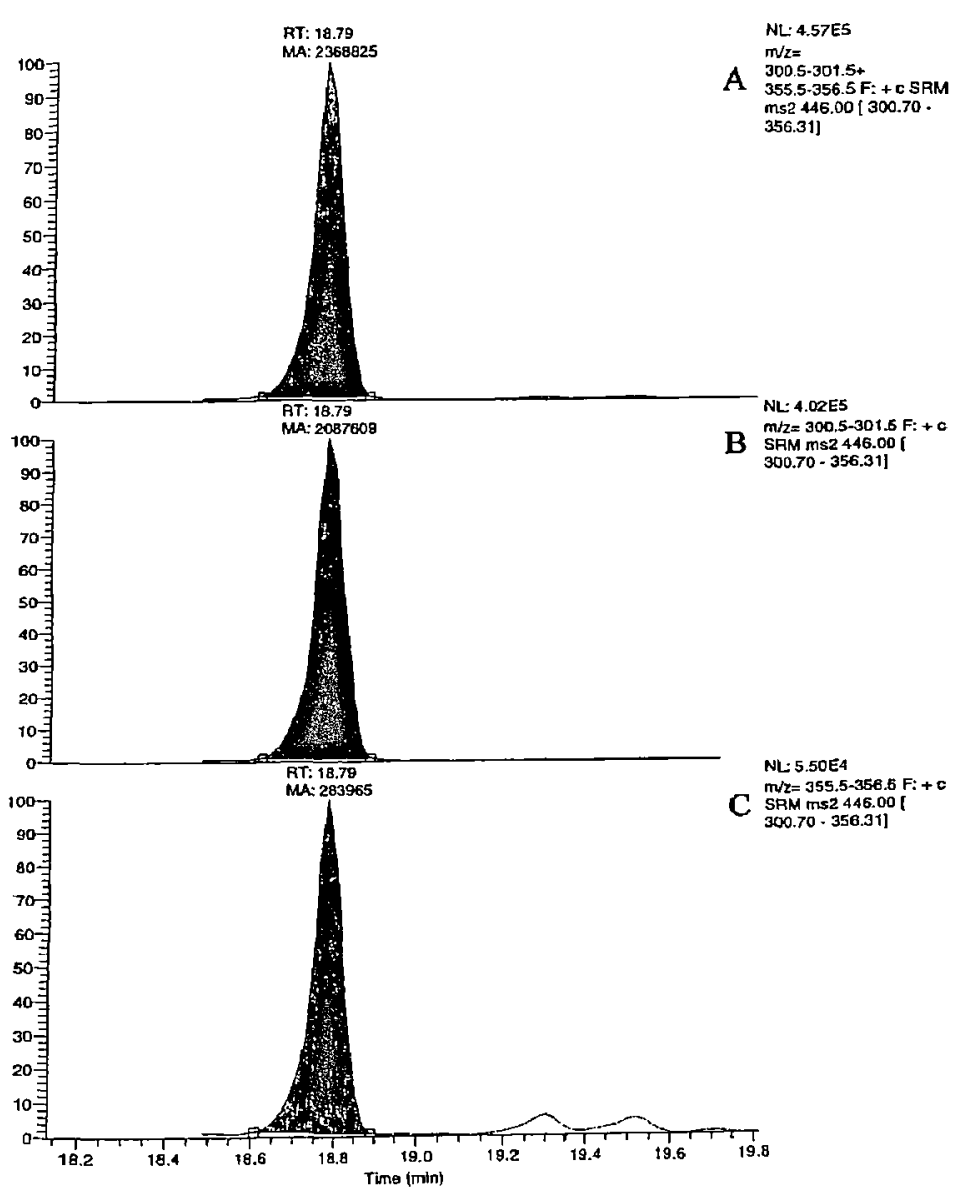

Figure 1 : Chromatogrammes d'un extrait de $100 \mathrm{mg}$ de poudre de poils de bovin trouvé positif pour la Méthyl Testostérone à la concentration de 3,4 pg/mg.

$A=$ chromatogramme représentant la somme des 2 ions fils de l'ion parent $\mathrm{m} / \mathrm{z} 446$

$B=$ ion fils $\mathrm{m} / \mathrm{z} 301 \quad C=$ ion fils $\mathrm{m} / \mathrm{z} 356$ 


\section{Application au contrôle du dopage chez l'homme}

L'application dans le dopage humain s'est faite dans le contexte judiciaire de trafic de substances illicites. Sur une série de 18 sportifs de deux équipes différentes, deux individus ont été reconnus comme consommateurs de stéroïdes. Le premier cas identifié l'était à la nandrolone avec $5,1 \mathrm{pg} / \mathrm{mg}$ (fig.2). L'analyse urinaire du même individu a donné des résultats négatifs (inférieurs à $0,05 \mathrm{ng} / \mathrm{ml}$ ) pour la norandrostérone (NA) et la noréthiocholanolone (NE), les métabolites urinaires de cette même nandrolone et de ses précurseurs. Il a été récemment prouvé qu'une voie endogène inconnue aboutit à ces métabolites, mais avec des seuils toujours inférieurs à $2 \mathrm{ng} / \mathrm{ml}$ d'urine [40-42]. Le second cas fait état de la détection d'undécanoate de testostérone à une concentration de $15,2 \mathrm{pg} / \mathrm{mg}$ (fig.3), bien que dans l'urine de ce même sportif le rapport testostérone/épitestostérone soit nettement inférieur à 6. Les deux sportifs ont reconnu spontanément leurs pratiques régulières du dopage après avoir pris connaissance de nos résultats.

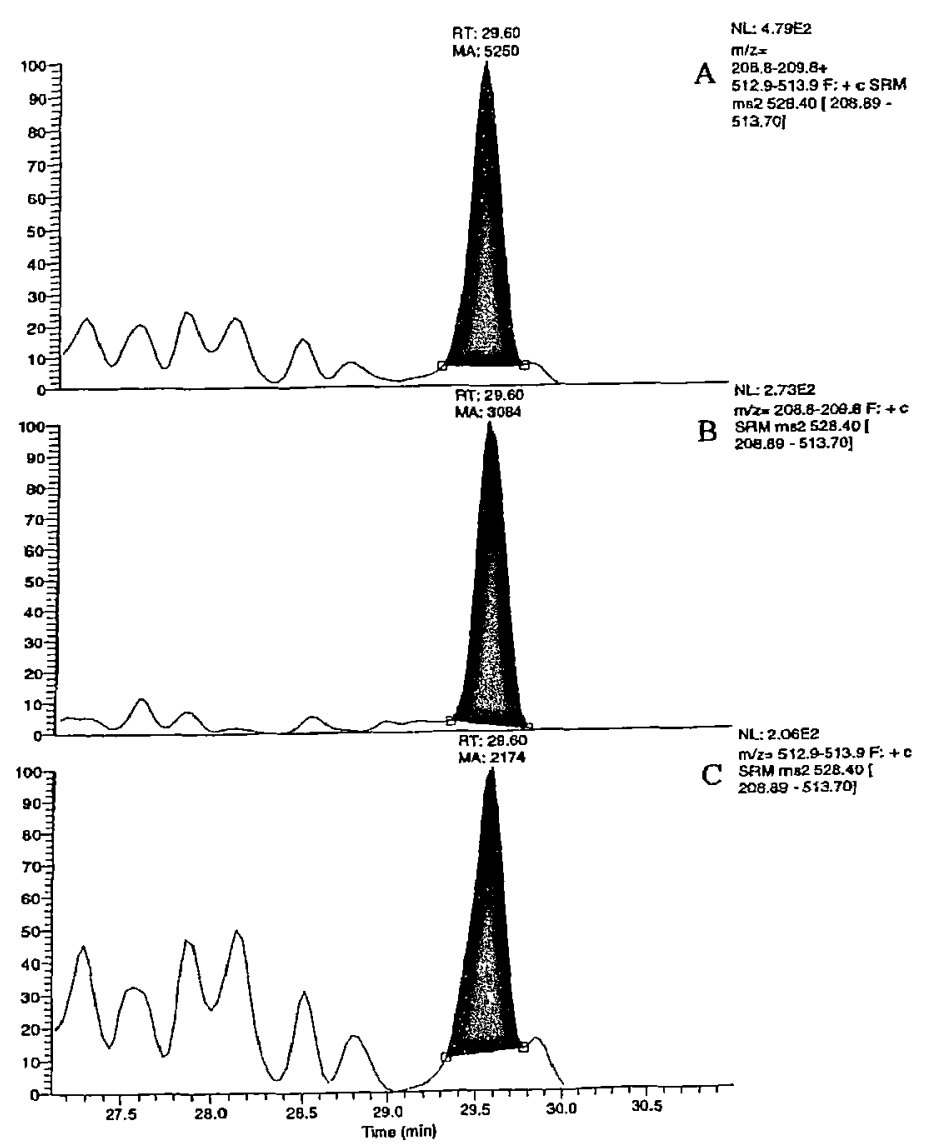

Figure 3: Chromatogrammes d'un extrait de $50 \mathrm{mg}$ de poudre de cheveux d'un sportif trouvé positif pour l'Undécanoate de Testostérone à une concentration de $15,2 \mathrm{pg} / \mathrm{mg}$.

$A=$ chromatogramme représentant la somme des 2 ions fils de l'ion parent $\mathrm{m} / \mathrm{z} 528$

$B=$ ion fils $\mathrm{m} / \mathrm{z} 209 \quad C=$ ion fils $\mathrm{m} / \mathrm{z} 513$
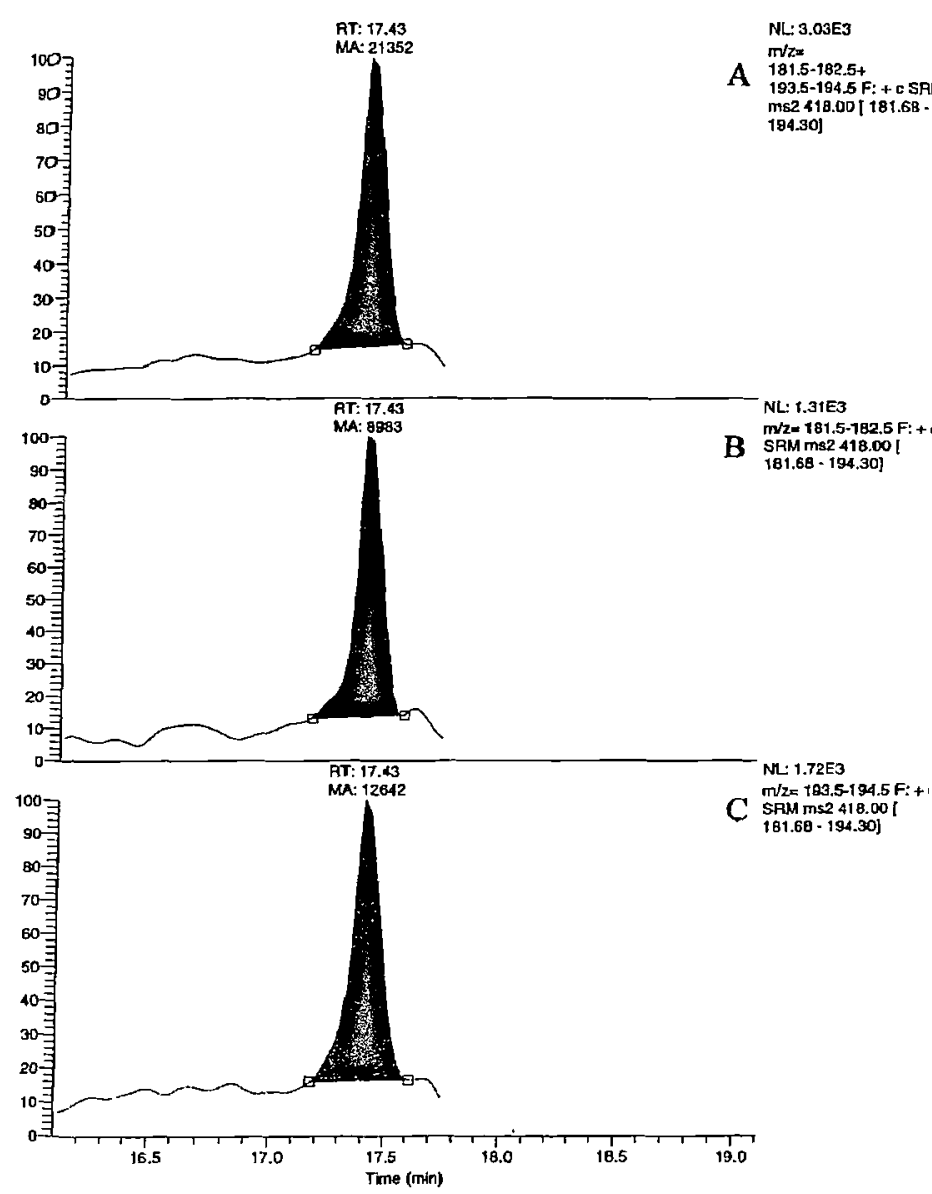

Figure 2 : Chromatogrammes d'un extrait de $50 \mathrm{mg}$ de poudre de cheveux d'un sportif trouvé positif pour la Nandrolone à une concentration de $5,1 \mathrm{pg} / \mathrm{mg}$.

$A=$ chromatogramme représentant la somme des 2 ions fils de l'ion parent $\mathrm{m} / \mathrm{z} 418$

$B=$ ion fils $\mathrm{m} / \mathrm{z} 182 \quad C=$ ion fils $\mathrm{m} / \mathrm{z} 194$

\section{Conclusion}

La technique de recherche des agents dopants anabolisants par CPG-SM-SM dans les phanères que nous avons validée et publiée [6] a montré son intérêt incontestable, en complément de l'urine, pour la recherche des agents dopants anabolisants que ce soit dans le domaine du contrôle de la production animale ou dans le domaine du contrôle du dopage chez le sportif.

Cependant une différence importante d'application existe entre ces deux domaines. En effet, en liaison avec nos travaux le ministère de l'Agriculture a publié deux techniques officielles de détection des $B$-agonistes et des anabolisants stéroïdiens dans les phanères et les utilisent. De plus l'interprofession a fait effectuer un grand nombre de contrôles aléatoires dans les poils des animaux avant abattage dans les différentes régions de France. Nous en avons réalisés plus de 1000 à ce jour. Pour l'instant, le ministère de la Jeunesse et des Sports n'a pas inclus en plus de l'urine, seul prélèvement biologique officiel actuel, ce milieu dans la loi $n^{\circ} 99-223$ du 23 mars 1999, mais nous espérons que d'autres 
milieux, dont les phanères, figureront dans les décrets d'applications à paraître. De plus à ce jour, aucun club, aucune fédération, ne nous a demandé, ni spontanément, ni suite à nos propositions d'effectuer des analyses sur ce milieu inaltérable, non falsifiable et qui permet une mise en évidence rétroactive de la prise d'agents dopants anabolisants. Par contre les Procureurs de la République et les Juges d'Instruction nous désignent régulièrement en cas de suspicion de prise d'agents dopants illégaux pour effectuer la recherche, l'identification et le dosage dans les phanères en plus de l'urine que ce soit chez l'homme ou chez les animaux de rente.

\section{Références}

1. Gaillard Y., Pépin G., 42ème Congrès Int. de Méd. Lég. et Méd. Soc., Lille, 9-11 septembre, 1998.

2. Debruykere G., Van Peteghem C.H.,de Sagher R., Influence of the consumption of meat contaminated with anabolic steroids on doping tests. Anal. Chim. Acta. $1993 ; 275: 49-56$.

3. Gaillard Y., Doucet F., Balland A., Pépin G., Détection of illegal clenbuterol use in calves using hair anlysis - Application in meat quality control. J. Chromatogr. B. 1997 ; $703:$ 85-95.

4. Marchand Ph., Maume D., Montrade M.P., Monteau F., LeBizec B., André F., Promoteurs de croissance dans le poil - Détection et identification d'agonistes $B$ adrénergiques par chromatographie gazeuse couplée à la spectrométrie de masse. Ministère de l'Agriculture, Document LDH/LNR/98A-p.2. 1998.

5. Prévost S., Marchand Ph., Gadé C., Monteau F., LeBizec B., André F., Promoteurs de croissance dans le poil - Détection et identification de stéroïdes anabolisants par chromatographie gazeuse couplée à la spectrométrie de masse. Ministère de l'Agriculture, Document LDH/LNR/98S-p.3. 1998.

6. Gaillard Y., Vayssette F., Balland A., Pépin G., Gas chromatographic-tandem mass spectrometric determination of anabolic steroids and theirs esters in hair - Application in doping control and meat quality control. J. Chromatogr. B. 1999 ; 735 (2) : 189-205.

7. Laure P., Médecins généralistes et dopage sportif : connaissances et attitudes. Santé Publique. $1997 ; 9$ (2) : 145-156.

8. de Mondenard J.P. Dictionnaire des Substances et Procédés Dopants en Pratique Sportive. Paris : Masson, $1991: 24$.

9. Franke W.W., Berendonk B., Hormonal doping and androgenization of athletes : a secret program of the German Democratic Republic government. Clin. Chem. 1997 ; 43 : 1262-1279.

10. de Mondenard J.P. Dictionnaire des Substances et Procédés Dopants en Pratique Sportive. Paris : Masson, 1991: 25.
11. Berendonke B. Doping Dokumente - Von der Forschung zum Betrug. Berlin : Springer-Verlag, 1991.

12. Franke W.W. Funktion und Instrumentalisierung des Sports in der DDR : Pharmakologische Manipulationen (Doping) und die Rolle der Wissenschaft. German Parliement Ed., 1995 ; 3/2 : 905.

13. Höppner M. Technik. Stasi report, $1977 ; 2: 243$.

14. Schäker W., Schubert K., Oettel M., Miedlich U., Gedrat J., Clausnitzer C., Bernstein B..In : Schäker W., ed. Zur Anwendung von Steroidsubstanzen (STS) im Training und Tiereexperiment sowie zur Qualitätsprüfung der STS-Präparate. Leipzig : FSF, 1991 : 1.

15. Breo D.L., Of MDs and muscles-lessons from two "retired steroid doctors". JAMA $1990 ; 263: 1697$, 1699, 1703-1705.

16. Voet W. Massacre à la chaine - Révélations sur 30 ans de tricheries. Paris : Calmann-Lévy, 1999.

17. Menthéour E. Secret défonce - Ma vérité sur le dopage. Paris : J.-C. Lattès, 1999.

18. Anonyme. Le Monde. 15 août 1998 : 14.

19. Delbeke F.T., Doping in cyclism : results of unannouced controls in Flanders (1987-1994). Int. J. Sports Med. $1996 ; 17: 434-438$.

20. de Mondenard J.P., Dictionnaire des Substances et Procédés Dopants en Pratique Sportive. Paris : Masson, 1991 : 139.

21. Anonyme. France Soir. 7 septembre $1998: 2$.

22. J.P. Bidet. L'Equipe. 8 septembre $1998: 3$

23. Yves Bordenave. Le Monde. 8 octobre $1998: 29$.

24. Yves Bordenave. Le Monde. 27 juillet $1998: 15$.

25. Gleixner A., Meyer H.H.D., Detection of estradiol and testosterone in hair of cattle by HPLC/EIA. Fresenius J. Anal. Chem. 1997 ; 357 : 1198-1201.

26. Gleixner A., Meyer H.H.D., Methods to detect anabolics in hair : use for food hygiene and doping control. Int. Lab., July $1998: 20$. 
27. Munoz-Guerra J., Carreras D., Soriano C., Rodriguez C., Rodriguez A.F., Use of ion trap gas chromatography-tandem mass spectrometry for detection and confirmation of anabolic substances at trace levels in doping analysis. J. Chromatogr. B 1997 ; 704 (1-2) : 129-141.

28. Schanzer W., Delahaut P., Geyer H., Machnik M., Horning S., Long-term detection and identification of metandienone and stanozolol abuse in athletes by gas chromatography-high-resolution mass spectrometry. J. Chromatogr. B 1996 ; 687 (1) : 93-108.

29. Shackleton C.H., Chuang H., Kim J., de la Torre X., Segura J., Electrospray mass spectrometry of testosterone esters : potential for use in doping control. Steroids $1997 ; 62$ (7) : 523529.

30. Thieme D., Grosse J., Sachs H, Mueller R.K., Detection of several anabolic steroids of abuse in human hair. In : Proceedings of the Manfred Donike 16th Cologne Workshop on Dope Analysis. Cologne : Sport und Buch, 1998 ; 9-31.

31. Kintz P., Cirimèle V., Sachs H., Jeanneau T., Ludes B., Testing for anabolic steroids in hair from two bobybuilders. Forensic Sci. Int. 1999 ; 101 : 209216.

32. Journal Officiel de la République Française, 24 mars 1999: 4399-4405.

33. Robbins C.R. Chemical et Physical Behavior of Human Hair. New-York : Springer, 1988.

34. Bhathnagar V., Anjaiah S., Puri N., Ramaihah A., $\mathrm{pH}$ of melanosomes of $\mathrm{B} 16$ murine melanoma is acidic : its physiological importance in the regulation of melanin biosynthesis. Arch. Biochem. Biophys. 1993 ; 307 : 183-192.
35. Howells L., Godfrey M., Sauer M.J., Melanin as an adsorbent for drug residues. 1994 ; 119 : 26912693.

36. Sachs H., Quality control by the Society of Hair Testing. Forensic Sci. Int. 1997 ; 84 (1-3) : 145-150.

37. Gaillard Y., Pépin G., Gas chromatographic-mass spectrometric quantification of dextropropoxyphene and norpropoxyphene in hair and whole blood after automated on-line solid-phase extraction Application in twelve fatalities. J. Chromatogr. B $1998 ; 709: 69-77$.

38. Leinonen A., Savonen L., Kuoppasalmi K., Improved purification of anaboolic steroids for GC/MS analysis. In : Proceedings of the Manfred Donike 12nd Cologne Workshop on Dope Analysis. Cologne : Sport und Buch, 1994 : 25-31.

39. Segura J., Ventura R., Jurado C., Derivatization procedures for gas chromatographic-mass spectrometric determination of xenobiotics in biological samples, with special attention to drugs of abuse and doping agents. J. Chromatogr. B $1998 ; 713$ : 61-90.

40. LeBizec B., Monteau F., Gaudin I., André F., Evidence for presence of endogenous 19-norandrosterone in human urine. J. Chromatogr. B 1999 ; $723: 157-172$.

41. Dehennin L., Bonnaire Y., Plou Ph., Urinary excretion of 19-norandrosterone of endogenous origin in man : quantitative analysis by gas chromatographymass spectrometry. J. Chromatogr. B $1999 ; 721$ (2) : 301-307.

42. Kintz P., Cirimèle V., Ludes B., Norandrostérone et norétiocholanolone : les métabolites révélateurs. Acta Clin. Belg. 1999 ; Suppl. 1 : 68-73. 